\title{
Psychometric Properties of the Chinese Breast Cancer Screening Beliefs Questionnaire
}

\section{Authorship}

(1) The conception and design of the study, or acquisition of data, or analysis and interpretation of data, (2) drafting the article or revising it critically for important intellectual content, (3) final approval of the version to be submitted.

Daniel Y.T. Fong, PhD School of Nursing, Li Ka Shing Faculty of Medicine, The University of Hong Kong $4^{\text {th }}$ floor, William M.W. Mong Block, 21 Sassoon Road, Pokfulam, Hong Kong (dytfong@hku.hk)

Cannas Kwok, PhD Sydney Nursing School, The University of Sydney

M02 - Sydney Nursing School, The University of Sydney, NSW, Australia (cannas.kwok@sydney.edu.au)

Kate White, PhD Sydney Nursing School, The University of Sydney

M02 - Sydney Nursing School, The University of Sydney, NSW, Australia (Kate.white@sydney.edu.au)

Corresponding author: Cannas Kwok, PhD Sydney Nursing School, The University of Sydney, M02 - Sydney Nursing School, The University of Sydney, NSW, Australia Phone: +61293510804

(cannas.kwok@sydney.edu.au). 


\section{Psychometric Properties of the Chinese Breast Cancer Screening Beliefs Questionnaire}

Background: Breast cancer is the most common type of cancer in women. The Chinese Breast Cancer Screening Beliefs (CBCSB) questionnaire was developed to measure ChineseAustralian women's beliefs, knowledge and attitudes about breast cancer and breast cancer screening.

Purpose: To assess the psychometrics of the modified version CBCSB in a Chinese-speaking community.

Methods: Two items in the original CBCSB were removed because they were not applicable to the Hong Kong setting, which resulted in an 11-item CBCSB. A total of 730 women aged at least 18 years old without a history of breast cancer self-completed the questionnaire. Results: Based on 730 Chinese-speaking women with mean age of 43 years, the three hypothesized subscales of the CBCSB had Cronbach's alpha ranging between 0.69 and 0.75. Non-responses to the items were at most only 3.3\%. The corrected item-total correlations for the hypothesized subscales ranged from 0.35 to 0.63 and were higher than those for the competing subscales. As hypothesized, the frequency of health practices was significantly associated with all subscales of the CBCSB. Confirmatory factor analysis showed an adequate fit for the hypothesized three-factor structure of the modified CBCSB questionnaire. Conclusions: The 11-item CBCSB questionnaire was culturally appropriate, reliable and valid in a Chinese-speaking community setting. It can be used to gain understanding of Chinese-speaking women's beliefs, knowledge and attitudes about breast cancer and breast cancer screening. It may also serve as an outcome for the development and assessment of public education programs for breast cancer screening. 
Keywords:

Breast cancer screening

Chinese

Psychometrics

Reliability

Validity 


\section{Psychometric Properties of the Chinese Breast Cancer Screening Beliefs Questionnaire}

\section{Introduction}

Breast cancer is the most common cancer in women across most, if not all, ethnic groups. In Hong Kong, breast cancer is the third leading cause of cancer deaths among women, accounting for 10.3\% of all cancer deaths in 2008 (Hospital Authority, 2011). Over the last two decades, the age-standardized incidence rate of female breast cancer has been steadily increased from 30.9 to 46.8 per 100,000 standard population (Hospital Authority, 2011).

Given that the causes of breast cancer remain largely unknown, early detection procedures, including breast self-examination, clinical breast examination, and mammography, are considerably important as secondary preventive measures. A previous meta-analysis showed that in particular, mammographic screening may reduce the risk for breast cancer-related death by 30\% (Leung et al., 2002). As a result, some Western countries, such as Australia and United Kingdom, have implemented national screening programs to provide mammograms for women in the target age group (Australian Cancer Council, 2011; NHS Cancer Screening Programmes, 2011). In Hong Kong, however, there is currently no population screening of breast cancer or official recommendation for breast cancer screening practices. The Well Women Clinics organized by the Tung Wah Group of Hospitals were the first and presently the largest comprehensive self-referred breast cancer screening program established in Hong Kong. Their services were provided on an out-of-pocket basis and health insurance does not cover the cost of a mammogram if it is requested for preventive purposes. In addition, there were also Breast Health Centres which perform breast cancer screening. They offered a fee waiver to low-income people whose income and asset do not exceed a predefined limit. 
Research has shown the attitudes and knowledge about breast cancer, and screening practices among Chinese women are generally unfavorable (Kwok and Sullivan, 2007; Tan et al., 2007). In addition, the concept of preventive health care has a low priority among the Chinese population in Hong Kong (Chua et al., 2005). In Hong Kong, 42\% of women refused to participate in annual mammography screening and clinical breast examination (Chua et al., 2005), and approximately 68\% of women had never had a mammogram (Yan, 2009). Moreover, in a Hong Kong women clinic where women were seeking care, the rate of routine breast self-examination was also as low as $44 \%$ (Abdullah and Leung, 2001). While there is a need to examine the attitudes, knowledge of, and barriers to breast cancer screening or specifically, to mammography (Chua et al., 2005; Yan, 2009), no studies had used an adequately tested instrument. An instrument is considered adequate if both its reliability and validity have been evaluated and found to meet certain criteria. Unfortunately, to our knowledge, there has been no adequately tested instrument for assessing the attitudes, knowledge of, and barriers to breast cancer screening in Hong Kong.

In response to this literature gap, the Chinese Breast Cancer Screening Beliefs (CBCSB) Questionnaire was originally developed to assess Chinese-Australian women’s beliefs, knowledge and attitudes towards breast cancer and screening practices. An initial pool of 32 items was developed from a comprehensive literature review in the field, and in-depth interviews conducted with Chinese-Australian women. The items were grouped under three dimensions: Attitudes towards general health check-ups (4 items), knowledge and perception about breast cancer (11 items), and mammographic screening practices (17 items). After careful assessment of the content validity, cultural appropriateness and psychometric performance, the CBCSB was reduced to 13 items loading onto three factors. The three factors represent three domains: 1) attitudes to general health checkups, which explore whether women engaged in general health checkups in the absence of signs and symptoms; 2) knowledge and perceptions about breast cancer, which explore cultural beliefs around breast 
cancer, and 3) barriers to mammographic screening, which explore personal and practical issues perceived by women to hinder their participation in breast screening. The 13-item CBCSB has been demonstrated to be reliable and valid in measuring the beliefs, knowledge and attitudes about breast cancer and breast cancer screening of 292 Chinese-Australian women. A detailed description of the development and psychometric test of the CBCSB can be found in Kwok et al. (2010).

However, the 13-item CBCSB was tested in a Chinese sample from an English-speaking community and may not be directly applicable in a Chinese-speaking community due to potential cultural differences. For instance, language was found to be a main barrier to mammography among Chinese-Australian women in Australia (Kwok et al., 2005). However, this item would not be applicable in a Chinese-speaking community in Hong Kong. Evaluating the cultural adaptability of the CBCSB in a Chinese-speaking community was thus desirable. Therefore, this study aimed to assess the psychometric performance of the modified CBCSB in Chinese-speaking women living in Hong Kong.

\section{Methods}

\section{Participants}

We planned to recruit 700 women who were at least 18 years old. However, those with a history of breast cancer or those who were unable to read traditional Chinese were excluded. A total of 31 centers of non-profit organizations covering different parts of Hong Kong participated in subject recruitment. Women who visited these centers were approached by research assistants to assess their study eligibility. All eligible women were invited to participate in the study. Those who were willing to participate were asked to sign an informed consent form before they completed a questionnaire. 
The planned sample size of 700 women was sufficient for a confirmatory factor analysis of the 11-item CBCSB questionnaire, using either the minimum requirement of 500 subjects or the rule of thumb of 20 subjects per item and allowing a small percentage of incomplete or problematic questionnaires (Comrey and Lee, 1992; Hair, 2010).

\section{Instruments}

A questionnaire was self-administered and included the following components: Modified CBCSB Questionnaire

The modified CBCSB questionnaire was derived from the original 13-item CBCSB developed for Chinese-Australians (Kwok et al., 2010). The two items in the "Barriers to mammographic screening” subscale of the original CBCSB questionnaire were removed: one item about the difficulty in arranging transportation to get a mammogram and the other item about the ability to speak English. These items were not applicable in Hong Kong, a city with an efficient public transportation system and everyone can get to and on public transportation. In particular, all public healthcare centers are accessible by public transportation. Moreover, most, if not all, practicing healthcare professionals in Hong Kong speak Cantonese. The resulting modified CBCSB questionnaire included 11 items in three subscales: attitudes towards general health check-ups (4 items); knowledge and perceptions about breast cancer (4 items); and barriers to mammographic screening (3 items). All of the items were rated on a 5-point Likert scale, with 1 corresponding to the lowest attitude, least knowledge or greatest barrier. A brief description of the items is provided in Fig. 1. For each subscale, the total score was standardized in the range between 0 and 100 for assessment, i.e. (total score minimum total)/(maximum total - minimum total). For instance, the score of a subscale of 4 items $=($ total score -4$) /(20-4)$. A higher subscale score indicated better attitude, more knowledge or lower barrier. 
Demographics and screening practices

The demographic information collected included age, marital status, education level, and employment status. Five items assessed the frequency of health check-up, such as the general health check-up, dental check-up, Pap smear, breast self-examination, clinical breast examination and mammography.

\section{Statistical analysis}

The demographic information and other clinical characteristics of the study participants were summarized using descriptive statistics. The modified CBCSB was scored identically to how the original version was scored (Kwok, et al., 2010). Case mean substitution was used for non-responded items within the subscale when participants provided at least $50 \%$ of valid responses to the other subscale items.

The modified CBCSB was first assessed for its item performance. Specifically, the internal reliability was assessed using Cronbach’s alpha. The correlated item-total correlations for the subscales were then examined. Moreover, the association between items and their competing subscales was assessed using the Spearman rank correlation coefficient.

The clinical validity was examined by using clinical criteria that should be associated with the CBCSB. First, we hypothesized that the subscale scores were positively associated with the frequency of health practices, including the general health check-up (0-4), dental check-up (0-4), Pap smear (0-4), breast self-examination (0-3), clinical breast examination (04) and mammogram (0-3), with 0 indicating that the health practice had never been practiced and a higher score indicating a more frequent practice. Second, we hypothesized that the education level (0-4) was positively associated with knowledge of breast cancer, as was shown in a previously study (Balogun, 2005). We performed linear regression analysis of a CBCSB subscale on a health practice to determine the extent to which the hypotheses were 
confirmed. A curved relationship was observed in two regression analyses, and thus, the quadratic terms were also estimated. Multiplicity due to multiple comparisons for the five health practices was accounted for by using the Holm’s procedure (Holm, 1979). The adequacy of a regression model was assessed by examining the standardized residuals.

Finally, a confirmatory factor analysis (CFA) was performed to examine the structural validity of the hypothesized three-factor structure of the modified CBCSB. Specifically, the CFA was performed using the polychoric correlation matrix of the CBCSB items, which accounted for the categorical nature of the item responses. The asymptotic covariance matrix was then computed, and a two-stage least-squared method was used to estimate the CFA parameters. The goodness-of-fit $\chi^{2}$ statistic and its degrees of freedom were reported. Due to the high sensitivity of the $\chi^{2}$ statistic to large samples, the goodness-of-fit assessment was based on the fit indices: the root mean square error of approximation (RMSEA), standardized root mean square residual (SRMR), comparative fit index (CFI), and non-normed fit index (NNFI). These addressed the parsimony correction, absolute fit, and comparative or incremental fit, as recommended (Hu and Bentler, 1998). They were used in tandem, and the CFA model was considered to be adequate if the RMSEA was close to 0.06 or lower, the SRMR close to 0.08 or lower, and the CFI and NNFI were close to 0.95 or greater (Hu and Bentler, 1999). The phrase “close to"' was used as suggested because of the fluctuation of the cutoff values under different modeling conditions and other fit indices used (Hu and Bentler, 1999). If there were doubts about inadequate fit, the addition of covariance was made based on the largest modification index (Brown, 2006).

SPSS (version 19, Armonk, New York) was used to conduct the general statistical analyses, and the R package (version 2.14.1, Vienna, Austria) was used to perform the CFA. A 5\% level of significance was used in all of the significance tests, and a 95\% confidence interval accompanied each estimate, where appropriate. 


\section{Results}

A total of 730 Chinese women in Hong Kong were recruited between May and October, 2009. Their demographics and frequency of health practices are summarized in Tables 1 and 2. The age of the women in the study covered a wide range, with a mean of 42.9 years (standard deviation [SD] = 11.7). Most of the women were married (68\%), had completed secondary school (59\%), or were employed (55\%). Only $44 \%$ of our study women had at least one mammographic screening, while most had a general health check-up (77\%), dental check-up (80\%), Pap smear (70\%) and breast self-examination (82\%) at least once. The missing values were those not filled out in the questionnaires. They are unlikely a result of ineligibility to the procedure because a woman could have responded as "Never" whether or not she considered herself as eligible to the procedures.

Table 3 summarizes the CBCSB subscales. There was only a mild skewness of the subscale scores, with floor effects of only $0.1 \%$ to $0.4 \%$ and ceiling effects of $1.0 \%$ to $4.8 \%$. Cronbach’s alpha of the three subscales ranged from 0.69 to 0.76 (Table 3). Missing responses accounted for $0.4 \%$ of all items on the "attitudes towards general health check-up" subscale, $2.9 \%$ to $3.3 \%$ of items in the "knowledge and perceptions about breast cancer" subscale, and there were no missing item responses in the "barriers to mammographic screening” subscale. The corrected item-total correlation for the three subscales, respectively, ranged from $0.38(\mathrm{Q} 4)$ to $0.61(\mathrm{Q} 1), 0.50(\mathrm{Q} 8)$ to $0.63(\mathrm{Q} 6)$, and $0.35(\mathrm{Q} 9)$ to $0.62(\mathrm{Q} 10)$. Details are shown in Table 4. All of the items correlated with their hypothesized subscales more than with the competing subscales.

The comparisons between the CBCSB and the frequency of health practices are described in Table 5. All of the health practices were positively associated with the "attitudes towards general health check-ups" subscale. The "knowledge and perceptions about breast 
cancer” subscale score was significantly higher in women who had more frequent general health check-ups. This score also increased with more frequent dental check-ups except in women who had dental check-ups at least once a year (Fig. 2(a)). Moreover, a higher education level corresponded to a $3.5(95 \% \mathrm{CI}=2.3$ to $4.7, \mathrm{p}<.001)$ increase in the scores for the knowledge subscale. The "barriers to mammographic screening” subscale score was significantly higher in women with more frequent health practices (Table 5), except for breast self-examination where the positive association was only evident in women who had a mammogram less than once each year (Fig. 2(b)). There was no deviation from the normality assumption in the regression analyses.

Based on the 730 women, the CFA of the three-factor hypothesized structure of the modified CBCSB resulted in the $\chi^{2}$ statistic $=294.7$ (degrees of freedom $=41, \mathrm{p}<.001$ ), RMSEA $=0.09(95 \%$ CI $=0.08$ to 0.10$)$, SRMR $=0.06$, CFI $=0.93$, and NNFI $=0.90$. The model fit was marginal, and the addition of covariance was considered. Based on the largest modification index, a covariance between items Q3 and Q4 was allowed and resulted in the $\chi^{2}$ statistic $=187.7($ degrees of freedom $=40, \mathrm{p}<.001), \mathrm{RMSEA}=0.07(95 \% \mathrm{CI}=0.06$ to 0.08), SRMR $=0.05, \mathrm{CFI}=0.96$, and NNFI $=0.94$. The corresponding standardized estimates are shown in Fig. 1 . All of the estimates were statistically significant $(p<.001)$.

\section{Discussion}

This study collected a large sample of Chinese-speaking women. The sample represents the target group of women who are eligible for breast cancer screening except for only a few women who were cohabitating. However, there was no evidence of differences in the attitudes, knowledge or barriers between women who were cohabitating and married women. Although our sample included young women who may not be recommended for mammographic screening, the CBCSB is deemed to be relevant because it aims to determine 
attitudes and beliefs towards breast cancer screening including mammography. For example, an item on the subscale related to barriers to mammographic screening is “I don't want to go for a mammogram because I would need to take off my clothes and expose my breasts” which is responded on a 5-Likert scale from "Strongly Disagree” to "Strongly Agree”. The statement asks about how much one perceive taking off clothes is a barrier to do mammogram. Thus, a younger adult woman should still be able to respond even though she may not have to do a mammogram at her age. Indeed, although younger adult women may not be recommended for mammographic screening, their current attitudes and beliefs may have an influence to their future practice. Therefore, it is desirable to have an instrument tested also on younger adult women. Hence, the sample had adequate coverage for evaluating the psychometric performance of the modified CBCSB.

The modified CBCSB had very small ceiling effects and negligible floor effects in all of the subscales. Moreover, the subscale scores had only mild skewness with small differences between the mean and the median. This eased the statistical analysis when the normal distribution assumption was required. The items of the instrument were well correlated with their hypothesized subscales, and they were less correlated with the competing subscales. Moreover, the instrument appeared to have good item consistency under each of the three subscales with no indication of overlap among the items. A good Cronbach’s alpha should be 0.7 or above and not much higher than 0.9 because a low Cronbach's alpha indicates a low degree of homogeneity among the subscale items, whereas a high value indicates item redundancy (Streiner and Norman, 2003). Both the "attitudes towards general health checkups” and "knowledge and perceptions about breast cancer” subscales had Cronbach’s alphas at approximately 0.75 , which was considered as good, although these Cronbach's alphas were slightly lower than the 0.79 reported in the Chinese-Australian population (Kwok et al., 2010). The Cronbach’s alpha of our three-item "barriers to mammographic screening” 
subscale was 0.69 , which was only slightly lower than the 0.70 reported for the five-item version in the Chinese-Australian population (Kwok et al., 2010). The removal of the two items did not substantially reduce the internal reliability of the subscale.

The modified CBCSB had good clinical validity with the associations between the three CBCSB subscales and the frequency of health practices, and the associations were consistent to those that were hypothesized. The "knowledge and perceptions about breast cancer” subscale was, however, not significantly associated with more frequent practice of breast self-examination, clinical breast examination and mammographic screening. The lack of significance with clinical breast examination was indeed also found among nurses in Singapore (Chong et al., 2002). Nevertheless, as hypothesized, the subscale was positively associated with education level.

Interestingly, the women who had mammographic screening at least once a year had more perceived barriers to mammographic screening. This finding was probably due to the limited representativeness of only 40 (8.2\%) women who had a mammogram once a year. Alternatively, women who had the most frequent mammographic screening had increased exposure to x-rays and may naturally think of the possible radiographic hazards. Thus, they may more likely perceive the potential hazards of mammogram and hence score higher in the “barriers to mammographic screening” subscale.

The hypothesized three-factor structure of the modified CBCSB was confirmed with only mild-to-moderate correlation among the three factors. The items loaded substantially on their hypothesized factors. Two items Q4 and Q9 loaded relatively smaller, though still of moderate size when compared with the usual acceptable level of 0.3 (Shevlin et al., 2000), on their respective factors. This was also observed in the factor loadings of the original CBCSB (Kwok, et al., 2010). On the other hand, there was small additional association between Q3 and Q4, both of which address whether seeking doctor consultations depended on the 
women's health condition. In general, a model fits better if it accounts more associations among the items. Therefore, accounting the additional association between Q3 and Q4 by addition of their covariance has improved the model fitness. The items were retained in the questionnaire despite their small association was, as they loaded at least a moderate amount on the subscale.

It is worthy to note that the CBCSB assesses the psychological impact of the participants. There can be other possible barriers related to financial support, support of healthcare and ancillary services such as insurance coverage, access to screening tests, and recommendation from healthcare professionals. However, the extent of concern that they may bring likely changes across different healthcare systems or regions. The CBCSB serves to psychologically assess beliefs, knowledge and attitudes towards breast cancer. Other potential barriers may be added where necessary according to a specific healthcare system or region.

The generalizability of the study results is limited to women who are able to read Chinese and self-complete questionnaires. In our study, the women who were unable to read traditional Chinese or possessed difficulty in completing the questionnaires were probably less educated or illiterate. This group of women may have different attitudes, knowledge and barriers when compared with other women. Moreover, administering the questionnaire by interview may produce different scores from those by self-completion. Further psychometric assessment for interview completion of the CBCSB is desirable. We have only assessed the traditional Chinese version of the CBCSB, which may not be applicable in Mainland China where simplified Chinese is the official written language. Further research on the cultural adaptation of the CBCSB in Mainland China is required. 


\section{Conclusion}

The modified CBCSB was shown to have good cultural adaptation for a Chinesespeaking community with a convenient public transportation system. We found evidence to support that the modified CBCSB questionnaire was reliable and that its hypothesized three subscales are valid for assessing Chinese women's beliefs, knowledge and attitudes about breast cancer and breast cancer screening. The instrument is essential for the development and assessment of public education programs for breast cancer screening. In addition, healthcare professionals may adopt the instrument to gain insights into the beliefs and needs of Chinese women about breast cancer and breast cancer screening. 


\section{References}

Australian Cancer Council, 2011. Cancer Council Australia.

http://www.cancer.org.au/home.htm. Accessed on 28th Nov, 2011.

Abdullah A.S., Leung T.Y., 2001. Factors associated with the use of breast and cervical cancer screening services among Chinese women in Hong Kong. Public Health 115(3), 212-217.

Balogun, M.O., 2005. Knowledge and Practice of Breast Self-Examination among Female Traders in Ibadan, Nigeria. Annals of Ibadan Postgraduate Medicine 3(2), 52-56.

Brown, T.A., 2006. Confirmatory factor analysis for applied research. Guilford Press , New York.

Chong, P.N., Krishnan, M., Hong, C.Y., Swah, T.S., 2002. Knowledge and practice of breast cancer screening amongst public health nurses in Singapore. Singapore medical journal 43(10), 509-516.

Chua, M.S., Mok, T.S., Kwan, W.H., Yeo, W., Zee, B., 2005. Knowledge, perceptions, and attitudes of Hong Kong Chinese women on screening mammography and early breast cancer management. The breast journal 11(1), 52-56.

Comrey, A.L., Lee, H.B., 1992. A first course in factor analysis, second ed. Hillsdale, New Jersey.

Hair, J.F., 2010. Multivariate data analysis, seventh ed. Prentice Hall, New Jersey.

Holm, S., 1979. A simple sequentially rejective multiple test procedure. Scandinavian Journal of Statistics 6, 65-70.

Hospital Authority, 2011. Hong Kong Cancer Registry.

Hu, L.T., Bentler, P.M., 1998. Fit indices in covariance structure modeling: Sensitivity to underparameterized model misspecification. Psychological Methods 3(4), 424-453. 
Hu, L.T., Bentler, P.M., 1999. Cutoff criteria for fit indexes in covariance structure analysis: conventional criteria versus new alternatives. Structural Equation Modeling 6(1), 1-55.

Kwok, C., Cant, R., Sullivan, G., 2005. Factors associated with mammographic decisions of Chinese-Australian women. Health education research 20(6), 739-747.

Kwok, C., Sullivan G., 2007. The concepts of health and preventive health practices of Chinese Australian women in relation to cancer screening. Journal of Transcultural Nursing 18(2), 118-126.

Kwok, C., Fethney, J., White, K., 2010. Chinese Breast Cancer Screening Beliefs

Questionnaire: development and psychometric testing with Chinese-Australian women. Journal of advanced nursing 66(1), 191-200.

Leung, G.M., Lam, T.H., Thach, T.Q., Hedley, A.J., 2002. Will screening mammography in the East do more harm than good? American journal of public health 92(11), 18411846.

NHS Cancer Screening Programmes, 2011. NHS Breast Screening Programme.http://www.cancerscreening.nhs.uk/breastscreen/index.html. Accessed on 28th Nov, 2011.

Shevlin M., Miles J.N.V. Davies M.N.O., Walker S. 2000. Coefficient alpha: a useful indicator of reliability? Personality and Individual Differences 28, 229-237.

Streiner, D.L., Norman, G.R., 2003. Health measurement scales: a practical guide to their development and use, third ed. Oxford University Press, Toronto.

Tan, S.M., Evans, A.J., Lam, T.P., Cheung, K.L., 2007. How relevant is breast cancer screening in the Asia/Pacific region? Breast 16(2), 113-119.

Yan, Y.Y., 2009. Breast Cancer: Knowledge and Perceptions of Chinese Women in Hong Kong. Global Journal of Health Science 1(2), 97-105. 


\section{Acknowledgments}

We thank Ms. Ada Chiu for her assistance with the data analysis.

Funding: This study was supported by a grant from the Cancer Institute NSW and Sydney Nursing School, University of Sydney.

\section{Conflict of Interest Statement}

There were no conflicts of Interest to disclose.

\section{Role of the funding source}

This study was supported by a grant from the Cancer Institute NSW and Sydney Nursing School, University of Sydney.

\section{Ethical approval}

The Institutional Review Board of the University of Hong Kong/Hospital Authority Hong Kong West Cluster (IRB No. UW 09-073). 
Fig. 1 Path diagram of a confirmatory factor analysis of the 11-item Chinese Breast Cancer

Screening Beliefs instrument in 730 Chinese women in Hong Kong. The values correspond to the standardized estimates and the model is considered of adequate goodness-of-fit.

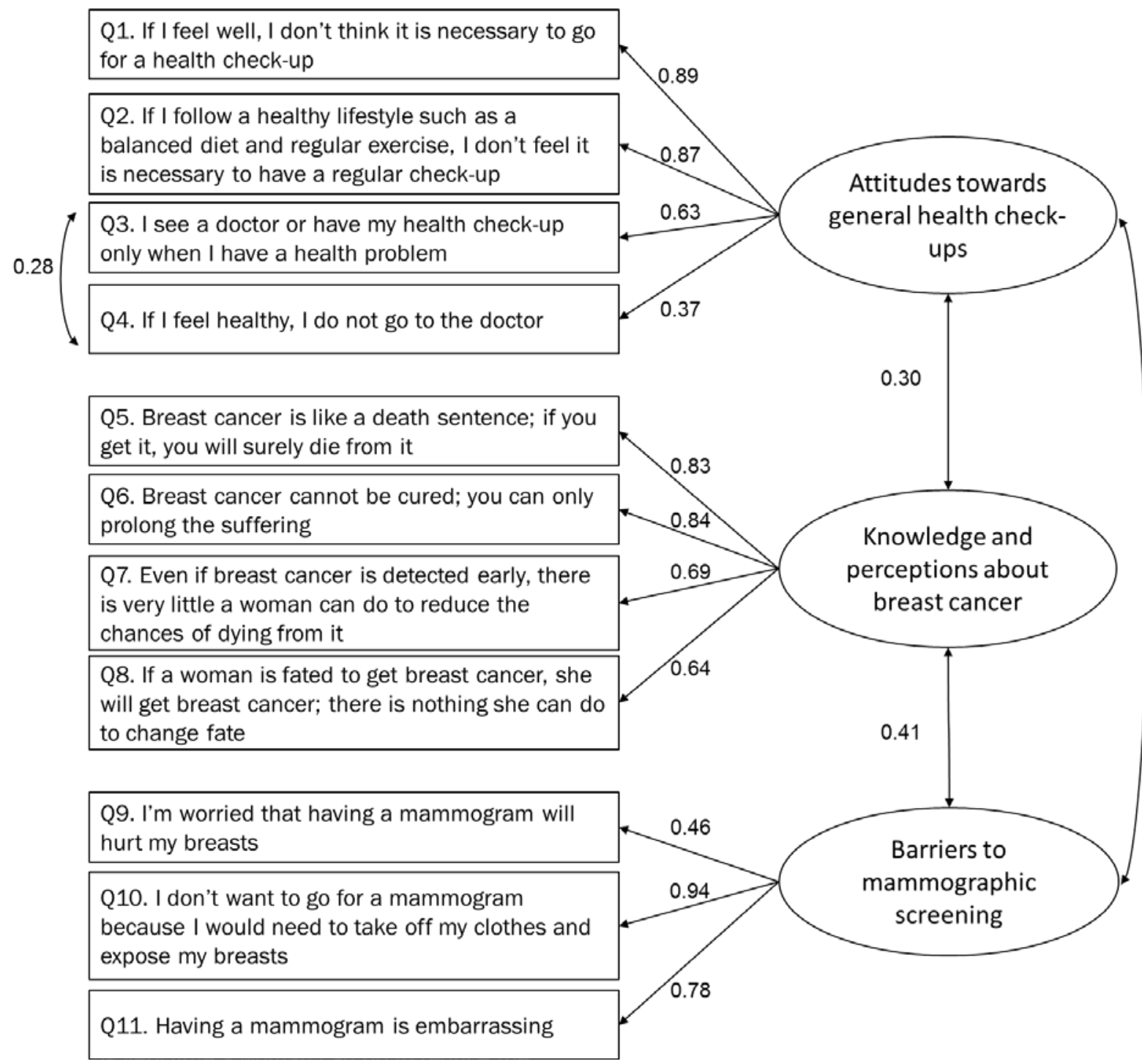


Fig. 2 The means and their 95\% confidence intervals (error bars) of (a) Knowledge and perceptions about breast cancer against the frequency of dental check-up; and (b) Barriers to mammographic screening against the frequency of having a mammogram. Note the quadratic relationships on the graphs were all statistically significant at 5\% level.

(a) Knowledge and perceptions about breast cancer against the frequency of dental check-up

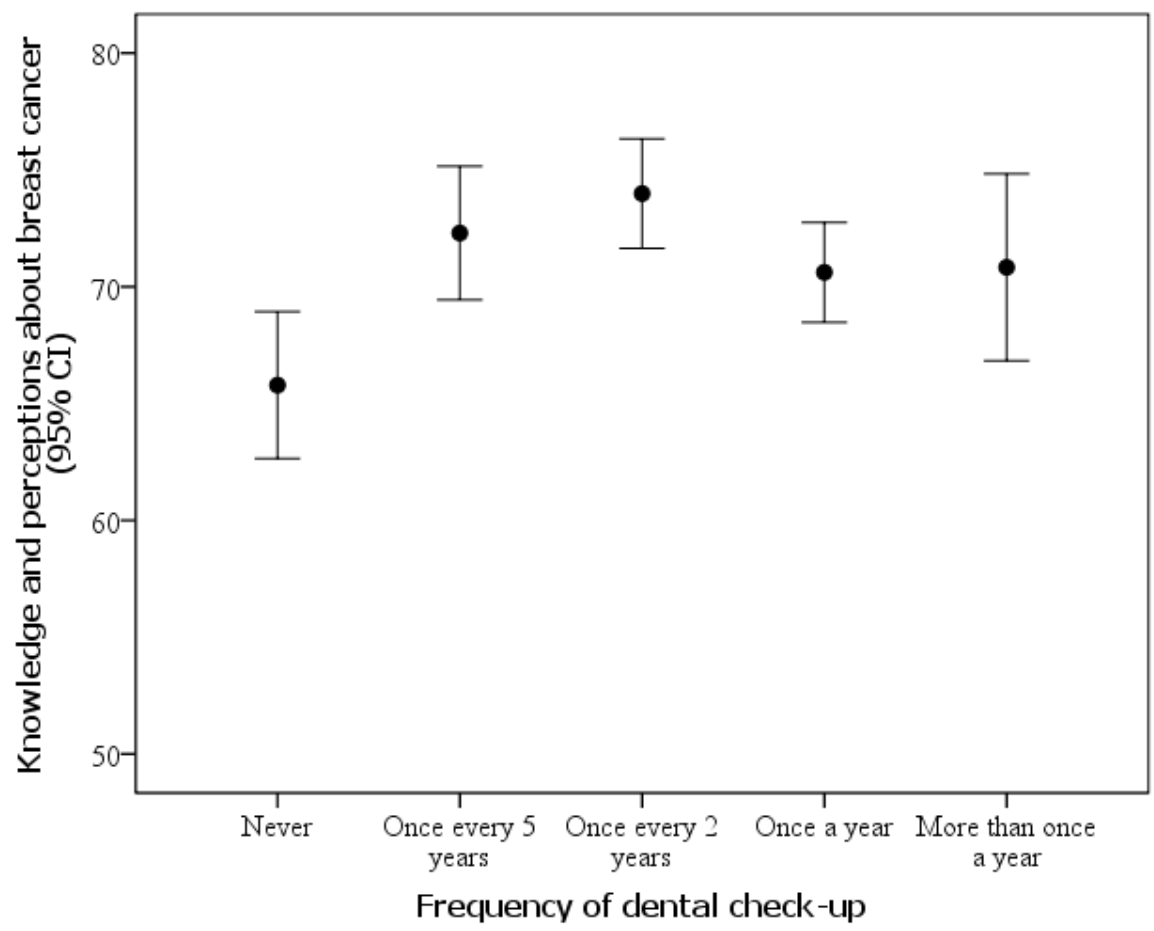


(b) Barriers to mammographic screening against the frequency of having a mammogram

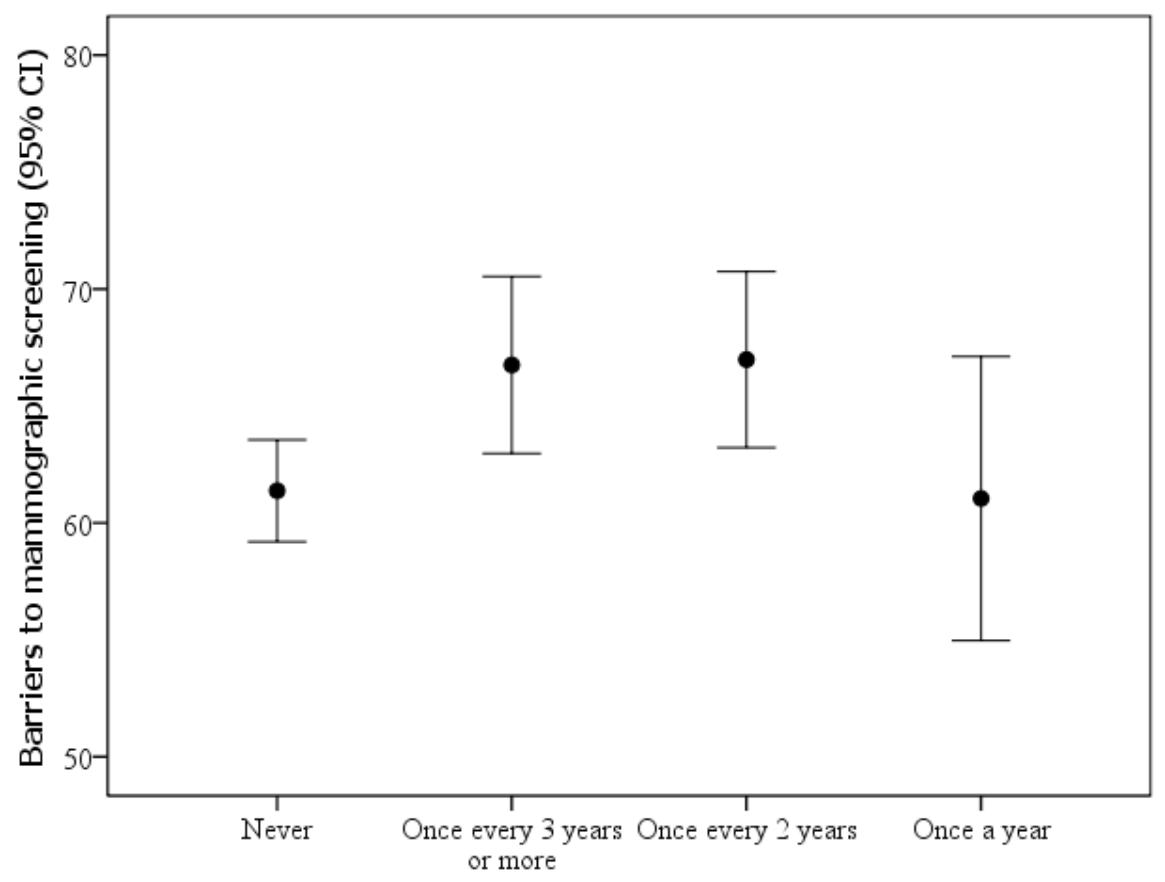

Frequency of having a mammogram 
Table 1.

Chinese Women Participants' Demographic Characteristics $(n=730)$.

\begin{tabular}{lcc}
\hline & $\mathrm{n}$ & $\%$ \\
\hline Age (years) (missing: $\mathrm{n}=71$ ) & \multicolumn{2}{c}{$42.9 \pm 11.7$} \\
$\quad$ Mean \pm standard deviation & \multicolumn{2}{c}{$18-80$} \\
$\quad$ Range & \multicolumn{2}{c}{} \\
Marital status (missing: $\mathrm{n}=7$ ) & 155 & 21.4 \\
$\quad$ Single & 492 & 68.0 \\
Married & 5 & 0.7 \\
Cohabited & 49 & 6.8 \\
Divorced/Separated & 22 & 3.0 \\
Widowed & & \\
Education level (missing: $\mathrm{n}=5$ ) & 10 & 1.4 \\
Never attended school & 133 & 18.3 \\
Primary school & 424 & 58.5 \\
Secondary school & 54 & 7.4 \\
Institutional training & 104 & 14.3 \\
Tertiary or above & & \\
Occupation (missing: $\mathrm{n}=$ 33) & 265 & 38.0 \\
Full time & 120 & 17.2 \\
Part time & 78 & 11.1 \\
Unemployed: seeking work & 102 & 14.6 \\
Unemployed: not seeking work & 106 & 15.2 \\
Retired & 24 & 3.4 \\
Housewife & 2 & 0.3 \\
Student & &
\end{tabular}




\section{Table 2.}

Chinese Women Participants' Health Practices $(n=730)$.

n

General health check-up (missing: $\mathrm{n}=24$ )

More than once a year

Once a year

Once every 2 years

Once every 5 years

Never

Dental check-up (missing: $\mathrm{n}=41$ )

More than once a year

70

Once a year

Once every 2 years

Once every 5 years

Never

Pap smear (missing: $\mathrm{n}=38$ )

More than once a year

Once a year

Once every 2 years

Once every 5 years

Never

Breast self-examination (missing: $\mathrm{n}=109$ )

At least once a month

Once every few months

Once a year

Never

Clinical breast examination (missing: $\mathrm{n}=243$ )

A year ago or less

More than a year and less than 2 years ago

86

2-3 years ago

69

9.5

More than 3 years ago

66

9.0

Never had one

84

11.5

Mammogram (missing: $\mathrm{n}=242$ )

Once a year

40

Once every two years

79

16.2

Once every three years or more

Never
95

274
$\%$

5.7

17.6

.5

Note: The missing values were those not filled out in the questionnaires. 
Table 3.

Subscale summary of the 11-item Chinese Breast Cancer Screening Beliefs instrument.

\begin{tabular}{|c|c|c|c|c|c|c|c|c|c|}
\hline $\begin{array}{l}\text { Subscale } \\
\text { (Number of items) }\end{array}$ & $\mathrm{n}$ & Mean & SD & Median & Minimum & Maximum & $\%$ floor & \% ceiling & $\begin{array}{l}\text { Cronbach's } \\
\text { alpha }\end{array}$ \\
\hline $\begin{array}{l}\text { Attitudes towards general health check- } \\
\text { ups (4) }\end{array}$ & 727 & 51.9 & 20.0 & 50.0 & 0 & 100 & 0.4 & 1.0 & 0.75 \\
\hline $\begin{array}{l}\text { Knowledge and perceptions about breast } \\
\text { cancer (4) }\end{array}$ & 709 & 70.8 & 15.8 & 75.0 & 0 & 100 & 0.1 & 4.8 & 0.76 \\
\hline Barriers to mammographic screening (3) & 730 & 61.7 & 18.5 & 66.7 & 0 & 100 & 0.3 & 3.3 & 0.69 \\
\hline
\end{tabular}




\section{Table 4.}

Corrected item-total correlation for the subscales of the 11-item Chinese Breast Cancer Screening Beliefs instrument.

\begin{tabular}{lccc}
\hline & & Subscale & \\
Item & Attitudes towards general & Knowledge and perceptions & Barriers to \\
health check-ups & about breast cancer & mammographic screening \\
\hline Q1 & 0.61 & 0.22 & 0.20 \\
Q2 & 0.59 & 0.19 & 0.17 \\
Q3 & 0.61 & 0.19 & 0.19 \\
Q4 & 0.38 & 0.14 & 0.14 \\
Q5 & 0.16 & 0.60 & 0.26 \\
Q6 & 0.16 & 0.64 & 0.21 \\
Q7 & 0.21 & 0.54 & 0.18 \\
Q8 & 0.18 & 0.50 & 0.27 \\
Q9 & 0.17 & 0.26 & 0.35 \\
\hline & 0.25 & 0.27 & 0.62 \\
\hline
\end{tabular}




\section{Table 5.}

Clinical validity of the 11-item Chinese Breast Cancer Screening Beliefs (CBCSB) instrument.

\begin{tabular}{|c|c|c|c|c|c|c|c|}
\hline \multirow[b]{2}{*}{ Health practice } & \multicolumn{3}{|c|}{$\begin{array}{l}\text { Attitudes towards general health } \\
\text { check-ups }\end{array}$} & \multicolumn{3}{|c|}{$\begin{array}{l}\text { Knowledge and perceptions about breast } \\
\text { cancer }\end{array}$} & $\mathrm{B}$ \\
\hline & $\mathrm{n}$ & $\begin{array}{c}\text { Unstandardized } \\
\text { coefficient } \\
(95 \% \mathrm{CI})\end{array}$ & p-value & $\mathrm{n}$ & $\begin{array}{c}\text { Unstandardized } \\
\text { coefficient } \\
(95 \% \text { CI })\end{array}$ & p-value & $\mathrm{n}$ \\
\hline General health check-up & 705 & $2.9(1.9,10.0)$ & $<0.001^{*}$ & 688 & $1.5(0.6,2.3)$ & $0.001^{*}$ & 706 \\
\hline Dental check-up & 688 & $3.3(2.2,4.4)$ & $<0.001^{*}$ & 671 & $\begin{array}{l}\text { Linear: } 6.2(3.2,9.2) \\
\text { Quadratic: }-1.4(-2.3,-0.6)\end{array}$ & $\begin{array}{l}<0.001 * \\
0.001 *\end{array}$ & 689 \\
\hline Pap smear & 691 & $3.0(1.9,4.1)$ & $<0.001 *$ & 675 & $0.7(-0.2,1.6)$ & 0.147 & 692 \\
\hline Breast self-examination & 619 & $2.9(1.5,4.2)$ & $<0.001 *$ & 619 & $1.3(0.2,2.4)$ & 0.020 & 621 \\
\hline $\begin{array}{l}\text { Clinical breast } \\
\text { examination }\end{array}$ & 484 & $2.6(1.5,3.7)$ & $<0.001 *$ & 484 & $-0.2(-1.0,0.7)$ & 0.732 & 486 \\
\hline Mammogram & 485 & $3.7(2.0,5.4)$ & $<0.001 *$ & 474 & $-1.2(-2.6,0.01)$ & 0.069 & 488 \\
\hline
\end{tabular}

Abbreviations: CI, confidence interval.

*Statistically significant at 5\% level of significance after adjustment for multiplicity by Holm’s procedure.

Note: An estimate corresponds to the estimated coefficient of a health practice in a regression analysis with a CBCSB subscale as the dependent variable. 